\title{
THE BEST CONSTANT IN AN INEQUALITY OF OSTROWSIKI TYPE
}

\author{
T. C. PEACHEY, A. MCANDREW AND S. S. DRAGOMIR
}

Abstract. We prove the constant $\frac{1}{2}$ in Dragomir-Wang's inequality [2] is best.

\section{Introduction}

The classical inequality of Ostrowski, $[1$, p. 469] is

Theorem 1.1. Let $I$ be an interval in $\mathbb{R}, I^{\circ}$ the interior of $I, f: I \rightarrow \mathbb{R}$ be differentiable on $I^{\circ}$. Let $a, b \in I^{\circ}$ with $a<b$ and $\left\|f^{\prime}\right\|_{\infty}=\sup _{t \in[a, i]}\left|f^{\prime}(t)\right|<\infty$. Then

$$
\left|f(x)-\frac{1}{b-a} \int_{a}^{b} f(t) d t\right| \leq\left[\frac{1}{4}+\frac{\left(x-\frac{a+b}{2}\right)^{2}}{(b-a)^{2}}\right](b-a)\left\|f^{\prime}\right\|_{\infty}
$$

for all $x \in[a, b]$.

The constant $\frac{1}{4}$ in (1.1) is the best possible.

For, suppose that

$$
\left|f(x)-\frac{1}{b-a} \int_{a}^{b} f(t) d t\right| \leq\left[k+\frac{\left(x-\frac{a+b}{2}\right)^{2}}{(b-a)^{2}}\right](b-a)\left\|f^{\prime}\right\|_{\infty}
$$

for all $x \in[a, b]$. Taking $f(x)=x$, gives $\left\|f^{\prime}\right\|_{\infty}=1$ and (1.2) becómes

$$
\left|x-\frac{a+b}{2}\right| \leq\left[k+\frac{\left(x-\frac{a+b}{2}\right)^{2}}{(b-a)^{2}}\right](b-a)
$$

for all $x \in[a, b]$. With $x=a$ this becomes

$$
\frac{b-a}{2} \leq\left(k+\frac{1}{4}\right)(b-a)
$$

giving $k \geq \frac{1}{4}$.

Received January 18, 1999.

1991 Mathematics Subject Classification. Primary 26D15, Secondary 26D99

Key words and phrases. Ostrowski inequality. 


\section{The Results}

In [2], Dragomir and Wang obtained a related inequality:

Theorem 2.1. Let $I, f, a, b$ be as above and $f^{\prime} \in L_{1}[a, b]$. Then

$$
\left|f(x)-\frac{1}{b-a} \int_{a}^{b} f(t) d t\right| \leq\left[\frac{1}{2}+\frac{\left|x-\frac{a+b}{2}\right|}{b-a}\right]\left\|f^{\prime}\right\|_{1}
$$

for all $x \in[a, b]$,

but did not prove that the constant $\frac{1}{2}$ is the best possible one.

In [3], S. S. Dragomir gave an extension of Theorem 2.1 for mappings with bounded variation, i.e., he proved the result:

Theorem 2.2. Let $f:[a, b] \rightarrow \mathbb{R}$ be a mapping with bounded variation on $[a, b]$. Then for all $x \in[a, b[$, we have the inequality:

$$
\left|f(x)-\frac{1}{b-a} \int_{a}^{b} f(t) d t\right| \leq\left[\frac{1}{2}+\frac{\left|x-\frac{a+b}{2}\right|}{b-a}\right] \bigvee_{a}^{b}(f)
$$

where $\bigvee_{a}^{b}(f)$ denotes the total variation of $f$ on $[a, b]$.

The constant $\frac{1}{2}$ is the best possible one.

For the sake of completeness and as the paper [3] is not published yet, we give here a. short proof of Theorem 2.2 .

Using the integration by parts formula for Riemann-Stieltjes integral, we have

$$
\int_{a}^{b} p(x, t) d f(t)=f(x)(b-a)-\int_{a}^{b} f(t) d t
$$

where

for all $x, t \in[a, b]$.

$$
p(x, t):=\left\{\begin{array}{l}
t-a \text { ift } \in[a, x) \\
t-b \text { ift } \in[x, b]
\end{array}\right.
$$

It is well known that if $p:[a, b] \rightarrow \mathbb{R}$ is continuous on $[a, b]$ and $v:[a, b] \rightarrow \mathbb{R}$ is with bounded variation on $[a, b]$, then

$$
\left|\int_{a}^{b} p(x) d v(x)\right| \leq \sup _{x \in[a, b]}|p(x)| \bigvee_{a}^{b}(v)
$$

Applying the inequality (2.4) for $p(x, \cdot)$ and $f$, we get

$$
\begin{aligned}
& \left|\int_{a}^{b} p(x, t) d f(t)\right| \leq \sup _{t \in[a, b]}|p(x, t)| \bigvee_{a}^{b}(f) \\
= & \max \{x-a, b-x\} \bigvee_{a}^{b}(f)=\left[\frac{b-a}{2}+\left|x-\frac{a+b}{2}\right|\right] \bigvee_{a}^{b}(f) .
\end{aligned}
$$


Using the identity (2.3), we deduce the desired result (2.2).

To prove the sharpness of the constant $\frac{1}{2}$ in the class of mappings with bounded variation, assume that the inequality (2.2) holds with a constant $C>0$, i.e.,

$$
\left|\int_{a}^{b} f(t) d t-f(x)(b-a)\right| \leq\left[C(b-a)+\left|x-\frac{a+b}{2}\right|\right] \bigvee_{a}^{b}(f)
$$

for all $x \in[a, b]$.

Consider the mapping $f:[a, b] \rightarrow \mathbb{R}$ given by

$$
f(x)=\left\{\begin{array}{l}
0 \text { if } x \in[a, b] \backslash\left\{\frac{a+b}{2}\right\} \\
1 \text { if } x=\frac{a+b}{2}
\end{array}\right.
$$

in (2.5). Then $f$ is with bounded variation on $[a, b]$ and

$$
\bigvee_{a}^{b}(f)=2, \quad \int_{a}^{b} f(t) d t=0
$$

and for $x=\frac{a+b}{2}$ we get in (2.5), $1 \leq 2 C$; which implies that $C \geq \frac{1}{2}$ and the theorem is completely proved.

Now, it is clear that if $f$ is differentiable on $(a, b)$ and $f^{\prime} \in L_{1}[a, b]$, then $f$ is with bounded variation on $[a, b]$ and applying Theorem 2.2 we get Theorem 2.1. But we are not sure that the constant $\frac{1}{2}$ is best in the class of differentiable mappings whose derivatives are in $L_{1}(a, b)$. We give an example showing that the constant $\frac{1}{2}$ remains best for this class of mappings, too.

Suppose that

$$
\left|f(x)-\frac{1}{b-a} \int_{a}^{b} f(t) d t\right| \leq\left[k+\frac{\left|x-\frac{a+b}{2}\right|}{b-a}\right]\left\|f^{\prime}\right\|_{1}, \quad x \in[a, b] .
$$

Let $C$ be any positive real and let

$$
f(x)=\frac{C}{C^{2}+x^{2}}-\tan ^{-1}\left(\frac{1}{C}\right)
$$

with $a=-1$ and $b=1$.

Direct calculation shows that $\int_{a}^{b} f(t) d t=0$.

Also, since $f^{\prime}(x) \leq 0$ for all $x \geq 0$,

$$
\begin{aligned}
\left\|f^{\prime}\right\|_{1} & =2 \int_{0}^{1}\left|f^{\prime}(t)\right| d t=-2 \int_{0}^{1} f^{\prime}(t) d t=2[f(0)-f(1)] \\
& =2\left[\frac{1}{C}-\frac{C}{C^{2}+1}\right]=\frac{2}{C\left(C^{2}+1\right)} .
\end{aligned}
$$


Substituting these into (2.6) and taking $x=0$ then gives

$$
\left|\frac{1}{C}-\tan ^{-1}\left(\frac{1}{C}\right)\right| \leq k \frac{2}{C\left(C^{2}+1\right)}
$$

so that

$$
k \geq \frac{C^{2}+1}{2}\left[1-C \tan ^{-1}\left(\frac{1}{C}\right)\right] .
$$

Since the right side tends to $\frac{1}{2}$ as $C \rightarrow 0+$, we get $k \geq \frac{1}{2}$, which shows that the constant $\frac{1}{2}$ is the best possible in Theorem 2.1 .

\section{References}

[1] D. S. Mitrinović, J. E. Pečarić and A. M. Fink, Inequalitite for Functions and Their Integrals and Derivatives, Kluwer Academic Publishers, Dordrecht, 1994.

[2] S. S. Dragomir and S. Wang, A new inequality of Ostrowski's type in $L_{1}$ anorm and applications to some special means and some numerical quadrature rules, Tamkang J. of Math. 28(1997), 239-244.

[3] S. S. Dragomir, On the Ostrowski's inequality for mappings with bounded variation and applications, RGMIA, Research Report Collection, 2(1999), 73-80.

School of Communications and Informatics, Victoria University of Technology, PO Box 14428, Melbourne City MC, Victoria 8001, Australia.

E-mail: tom, amca, sever@matilda.vut.edu.au 